\title{
NODAL QUINTIC THREEFOLDS AND NODAL OCTIC SURFACES
}

\author{
CIPRIAN BORCEA
}

(Communicated by Louis J. Ratliff, Jr.)

\begin{abstract}
The relation between the nodes and the defect of nodal quintic threefolds or double solids ramified over nodal octic surfaces has implications on the existence of such varieties with fewer nodes.
\end{abstract}

\section{INTRODUCTION}

Consider the space $\prod_{n}^{d}=P\left(H^{0}\left(P_{n}, \mathscr{O}_{P_{n}}(d)\right)\right)$ of hypersurfaces of degree $d$ in $P_{n}$, the projective $n$-dimensional space (over $C$ ). Clearly $\prod_{n}^{d}=P_{N}$, with $N=\left(\begin{array}{c}n+d \\ d\end{array}\right)-1$. The points in $\prod_{n}^{d}$ corresponding to singular hypersurfaces build up the so-called "discriminant" hypersurface $\Delta \subset \prod_{n}^{d}$, which is of degree $(d-1)^{n}(n+1)$ and is naturally identified with the dual variety of the Veronese embedding of $P_{n}$ by $\mathscr{O}_{P_{n}}(d)$ (cf. [2]).

The smooth part of $\Delta$ consists of those hypersurfaces in $P_{n}$ which have no other singularity but one node, i.e. an ordinary double point. A small deformation of a node being either a node or a smooth point, the set $\mathscr{N} \subset \prod_{n}^{d}$ which parametrizes nodal hypersurfaces of degree $d$ in $P_{n}$ (i.e. hypersurfaces with at most nodes as singularities) is open and contains the smooth part of $\Delta$. In fact, the divisor $\mathscr{N} \cap \Delta$ of $\mathscr{N}$ may be decomposed into a disjoint union of locally closed subvarieties

$$
\mathscr{N} \cap \Delta=\bigcup_{\nu \geq 1} \mathcal{N}_{\nu}
$$

where $\mathscr{N}_{\nu}$ parametrizes nodal hypersurfaces with precisely $\nu$ nodes. There are a priori upper bounds for the number of singularities on nodal hypersurfaces [20], so that the union above is actually finite.

Nodal varieties are particularly interesting in dimension three, due to the fact that three-dimensional nodes allow "small resolutions" [1]. This brings to the fore not only the case of nodal threefolds in $P_{4}$, but also that of nodal surfaces in $P_{3}$ of even degree, since one may consider double coverings ramified over such surfaces (cf. [4, 10, 22]).

Received by the editors September 11, 1989 and, in revised form, November 10, 1989.

1980 Mathematics Subject Classification (1985 Revision). Primary 14J30, 14J25. 
Besides the number of nodes $\nu$, another important invariant $\delta$, which Clemens called defect, attaches to a nodal threefold $X$; namely,

$$
\delta(X)=b_{4}(X)-b_{2}(X),
$$

where $b_{k}$ denotes the $k$ th Betti number. If $X$ deforms, but maintains its number of nodes, $\delta$ remains constant.

Now specialize further to the case of nodal (projective) threefolds with trivial dualizing sheaf, a case also relevant for physicists (cf. [18]), at least insofar as projective small resolutions with small Euler number (e.g. \pm 6 , but not 0 ) might be produced. Then $\delta(X)$, which is reflected in the homology of a small resolution $\widehat{X}$, or, for that matter, of the standard resolution $\tilde{X}$, will also be reflected in the (infinitesimal) deformation theory of such a resolution, since:

$$
\begin{aligned}
h^{1}\left(\widehat{X}, \Theta_{\widehat{X}}\right) & =h^{1}\left(\widehat{X}, \Omega_{\widehat{X}}^{2}\right)=\frac{1}{2}\left(b_{3}(\widehat{X})-2\right) \\
h^{1}\left(\tilde{X}, \Theta_{\widetilde{X}}\right) & =h^{1}\left(\widetilde{X}, \Omega_{\widetilde{X}}^{2}\right)=\frac{1}{2}\left(b_{3}(\widetilde{X})-2\right)
\end{aligned}
$$

(cf. [6, 17]). This basically means that, in the versal deformation of $X$, the locus along which the number of nodes remains $\nu(X)$ has to be smooth. Explicitly, we have:

Theorem A. Let $\mathscr{N} \subset \prod_{n}^{d}$ denote the space of nodal quintic threefolds ( $n=4$, $d=5)$ or nodal octic surfaces $(n=3, d=8)$. The connected components of the locally closed subvarieties $\mathscr{N}_{\nu}$, parametrizing hypersurfaces with $\nu$ nodes, are smooth.

Further, we address the question: What is the actual range of $\nu$ ?

We shall prove:

Theorem B. Let $X$ be a nodal quintic threefold (resp. a double solid ramified over a nodal octic surface), with $\nu(X)$ nodes and defect $\delta(X)$. Then the range of $\nu$ over $\mathscr{N} \subset \Pi_{4}^{5}$ (resp. $\prod_{3}^{8}$ ) omits at most $\delta(X)$ integers in the interval $[0, \nu(X)]$.

Concrete estimates of this nature are given in $\S 4$. As an illustration, we mention:

Lemma 4.2.4. The dependency locus of two generic sections in the HorrocksMumford bundle over $P_{4}$ is a quintic with 100 nodes and defect $\delta=3$.

A question raised by Schoen in [17] is also answered.

We now proceed with the details.

\section{Nodes AND RESOlutions}

A node is a nondegenerate critical point of a complex analytic function. Thus, by the Morse-lemma a node in dimension $n$ is analytically equivalent with the hypersurface germ defined by $x_{0}^{2}+x_{1}^{2}+\cdots+x_{n}^{2}=0$ in $0 \in C^{n+1}$, which is a 
cone over the projective smooth quadric $Q_{n-1}$ (in the projective space $P_{n}$ at infinity). The versal deformation of this germ is given by:

$$
x_{0}^{2}+x_{1}^{2}+\cdots+x_{n}^{2}+t=0, \quad \text { with } t \in(C, 0) \text {. }
$$

The standard resolution morphism may be identified with the blow-down of the zero-section in $\mathscr{O}_{Q_{n-1}}(-1)$, which is the line bundle on $Q_{n-1}$ obtained by restricting the tautological bundle of $P_{n}$. The exceptional set is $Q_{n-1}$.

Now, $n=3$ is the only case in which $Q_{n-1}$ admits nontrivial fiberings, as a consequence of the isomorphism $Q_{2} \approx P_{1} \times P_{1}$. In this case, the exceptional divisor $Q_{2}$ in the standard resolution may be blown-down to any of its two factors $P_{1}$, yielding the so-called "small" resolutions. Although both of these resolutions are locally equivalent with the blow-down of the zero-section in $\mathscr{O}_{P_{1}}(-1) \oplus \mathscr{O}_{P_{1}}(-1)$, in a global context the two processes may produce nonisomorphic results. Moreover, the question whether a nodal projective threefold remains projective after small resolutions of its nodes is a delicate one. It is dealt with in [22].

\section{THE DEFECT}

2.1. For double solids ramified over nodal surfaces of even degree $d \geq 4$, Clemens [4] gave the following formula for computing the defect:

Let $S=S_{2}(d)$ be the ramification surface in $P_{3}$, with singular locus $N$ consisting of $\nu$ nodes.

Let $V=V_{3 d / 2-4}(N)$ denote the space of homogeneous polynomials of degree $(3 d / 2-4)$ in $P_{3}$, vanishing on $N$.

Then:

$$
\operatorname{dim} V=\left(\begin{array}{c}
3 d / 2-1 \\
3
\end{array}\right)-\nu+\delta .
$$

2.2. For nodal threefolds in $P_{4}$, Werner [22], using [15], gave the analogous result:

Let $T=T_{3}(d)$ be a degree $d \geq 3$ hypersurface in $P_{4}$, with singular locus $N$ consisting of $\nu$ nodes.

Let $W=W_{2 d-5}(N)$ denote the space of homogenous polynomials of degree $(2 d-5)$ in $P_{4}$, vanishing on $N$.

Then:

$$
\operatorname{dim} W=\left(\begin{array}{c}
2 d-1 \\
4
\end{array}\right)-\nu+\delta .
$$

2.3. Now, if the nodal double solid (resp. threefold in $P_{4}$ ) is supposed to have trivial canonical sheaf, i.e. we restrict ourselves to the case of nodal octic surfaces (resp. quintic threefolds), a basic observation is that $P(V)$ (resp. $P(W)$ ) has the further significance of being the projective tangent space in $S \in \prod_{3}^{8}$ (resp. $T \in \Pi_{4}^{5}$ ) to the (germ) locus $\mathscr{N}_{\nu}$ where $S$ (resp. $T$ ) deforms locally in a trivial manner (in the sense of $[5,9]$ ), i.e. maintains $\nu$ nodes. This is an immediate consequence of the versal deformation formula for a node. 
But a deformation of $S$ (resp. $T$ ) in which the nodes deform trivially preserves (by an Ehresmann-type argument) the topological type and hence the defect.

Note that $\mathscr{N}_{\nu}$ is reduced, since a small resolution of a projective nodal threefold with trivial canonical sheaf has unobstructed deformation space [19], while our hypersurfaces belong to complete families which, modulo the action of the linear projective group, become universal at the points considered. We also use here the fact that a small deformation of a nodal double solid is a nodal double solid (cf. [21, Theorem 3.9] and 2.4. below).

This yields our:

Theorem A. Let $\mathscr{N} \subset \prod_{n}^{d}$ denote the space of nodal octic surfaces $(n=3, d=$ $8)$ or nodal quintic threefolds $(n=4, d=5)$. The connected components of the locally closed subvarieties $\mathcal{N}_{\nu}$, parametrizing hypersurfaces with $\nu$ nodes, are smooth.

Such a connected component will be called of type $(\nu, \delta)$, according to the corresponding defect $\delta$, which may well vary in case $\mathscr{N}_{\nu}$ has several components.

2.4. A different line of argument extends the conclusion of Theorem $A$ to the cases $(n, d)=(3,6),(3,4),(4,4)$, and $(4,3)$, i.e. those producing Fano nodal threefolds. This uses the theory of (embedded) locally trivial deformations, as developed in [9].

Let $f: X \hookrightarrow S$ denote the inclusion of a Fano nodal hypersurface $X$ in $P_{4}=S$ or of a Fano nodal double solid $X$ in $P\left(\mathscr{O}_{P_{3}}(d / 2) \oplus \mathscr{O}_{P_{3}}\right)=S$.

One has an exact sequence of tangent cohomology sheaves:

$$
0 \rightarrow \mathscr{T}_{X}^{0} \rightarrow f^{*} \mathscr{T}_{S}^{0} \rightarrow \mathscr{T}_{X / S}^{1} \rightarrow \mathscr{T}_{X}^{1} \rightarrow 0 .
$$

Put: $\mathscr{N}_{X / S}^{\prime}=\operatorname{Ker}\left(\mathscr{T}_{X / S}^{1} \rightarrow \mathscr{T}_{X}^{1}\right)$.

The vanishing of $H^{1}\left(\mathscr{N}_{X / S}^{\prime}\right)$ ensures the smoothness of the germ parametrizing the locally trivial deformations of $X$ in $S$ (cf. [9, Proposition 2.3]).

In our case, $H^{1}\left(\mathcal{N}_{X / S}^{\prime}\right)=0$ follows from $H^{2}\left(\mathscr{T}_{X}^{0}\right)=0$, which is proved in [6, Theorem 4.1 with 3.4] and $H^{1}\left(f^{*} \mathscr{T}_{S}^{0}\right)=0$, obtained from the exact sequence:

$$
0=H^{1}\left(\mathscr{T}_{S}^{0}\right) \rightarrow H^{1}\left(f^{*} \mathscr{T}_{S}^{0}\right) \rightarrow H^{2}\left(\mathscr{T}_{S}^{0} \otimes \mathscr{O}_{S}(-X)\right) \rightarrow H^{2}\left(\mathscr{T}_{S}^{0}\right)=0
$$

and the vanishing of $H^{2}\left(\mathscr{T}_{S}^{0} \otimes \mathscr{O}_{S}(-X)\right)$. For the latter, we may suppose $X$ smooth and use the normal bundle exact sequence:

$$
H^{0}\left(\mathcal{N}_{X / S}\right) \rightarrow H^{1}\left(\mathscr{T}_{X}^{0}\right) \rightarrow H^{1}\left(f^{*} \mathscr{T}_{S}^{0}\right) \rightarrow H^{1}\left(\mathcal{N}_{X / S}\right)=0
$$

where the first arrow is surjective by completeness of the family to which $X$ belongs.

Furthermore, as shown in [9], the vanishing of $H^{1}\left(\mathcal{N}_{X / S}^{\prime}\right)$ also implies that any local deformation of the multigerm $(X, \operatorname{Sing}(X))$ is induced by a global 
embedded deformation of $X$ in $S$; in particular, Theorem B improves to the statement that any number of nodes, below $\nu(X)$, can be obtained.

For constructions of nodal sextic surfaces with up to 64 nodes, see [3].

For nodal quartic surfaces, the largest number of nodes: 16 , is realized by Kummer surfaces in $P_{3}$.

Nodal quartic threefolds may have up to 45 nodes, while for cubic threefolds the bound is: 10 nodes (cf. $[6,22])$.

\section{ON THE NUMBER OF NODES}

To simplify notation, we shall pursue the case of nodal quintic threefolds, that of nodal octic surfaces being clearly parallel.

3.1. Note that for quintics, the dimension of a component of type $(\nu, \delta)$ is

$$
\operatorname{dim} P(W)=\left(\begin{array}{l}
9 \\
4
\end{array}\right)-\nu+\delta-1=125-\nu+\delta .
$$

3.2. Put $\rho=\nu-\delta$ and note that, given a nodal quintic threefold $X$ of type $(\nu, \delta)$, with nodes at $x_{j} \in P_{4}, 1 \leq j \leq \nu, \rho$ is the rank of the matrix $\left(q_{i}\left(x_{j}\right)\right)_{i, j}$, where $\left(q_{i}\right)_{1 \leq i \leq 126}$ denotes a basis in the space of quintic homogeneous polynomials.

3.3. Like smooth quintics, nodal quintic threefolds do not admit a continuous group of automorphisms. Indeed, this would manifest itself in $h^{0}\left(\widehat{X}, \Theta_{\widehat{X}}\right)$, where $\widehat{X} \rightarrow X$ is a small resolution and $\Theta_{\widehat{X}}$ the tangent bundle. Since $\widehat{X}$ has trivial canonical bundle $\Theta_{\widehat{X}}=\Omega_{\widehat{X}}^{2}$.

Let $X_{0}$ denote a smooth quintic threefold with Euler number $e_{0}=-200$ and number of moduli $m_{0}=h^{1}\left(\Theta_{X_{0}}\right)=1-e_{0} / 2=101$.

Recall [4, 22] that:

$$
\begin{aligned}
b_{1}(\hat{X}) & =b_{1}(X)=0, \\
b_{2}(\widehat{X}) & =b_{4}(\widehat{X})=b_{4}(X)=1+\delta, \\
b_{3}(\widehat{X}) & =b_{3}(X)-\nu+\delta, \\
e(\widehat{X}) & =e_{0}+2 \nu .
\end{aligned}
$$

With obvious shorthand, and using the Hodge decomposition for Moisheson manifolds, we have:

$$
\begin{aligned}
& \chi\left(\Theta_{\widehat{X}}\right)=\frac{1}{2} e=\frac{1}{2} e_{0}+\nu, \\
& m:=h^{1}\left(\Theta_{\widehat{X}}\right)=h^{21}=\frac{1}{2} b_{3}(\widehat{X})-1=m_{0}-\nu+\delta=m_{0}-\rho, \\
& h^{2}\left(\Theta_{\widehat{X}}\right)=h^{22}=h^{11}, \\
& h^{3}\left(\Theta_{\widehat{X}}\right)=h^{23}=\frac{1}{2} b_{5}(\widehat{X})=0, \\
& h^{02}+h^{11}+h^{20}=b_{2}(\widehat{X})=1+\delta .
\end{aligned}
$$

Therefore $h^{02}=h^{20}=h^{0}\left(\Theta_{\widehat{X}}\right)=0$, that was to be proven. 
3.4. We have already encountered the relation:

$$
\rho=m_{0}-m=101-m \leq 101
$$

with $m=h^{1}\left(\Theta_{\widehat{X}}\right)$ giving the number of moduli of a small resolution of $X$, which, as mentioned in the introduction, is also the number of moduli of the standard resolution $\tilde{X}$ of $X$.

Let $\left(\mathscr{N}_{\nu}, X\right)$ denote the (germ of the) connected component of $\mathscr{N}_{\nu}$ to which $X$ belongs.

Our discussion shows in particular that the versal deformation of $\widehat{X}$ (resp. $\tilde{X}$ ) may be produced by small (resp. standard) resolutions of the nodal quintics lying on a slice of $\left(\mathcal{N}_{\nu}, X\right)$ transversal to the orbit of $X \operatorname{under} \operatorname{Aut}\left(P_{4}\right)=$ PGL(5). Indeed:

$$
m=\operatorname{dim}\left(\mathcal{N}_{\nu}, X\right)-\operatorname{dim} \operatorname{Aut}\left(P_{4}\right) .
$$

3.5. Return now to the matrix $\left(q_{i}\left(x_{j}\right)\right)_{i, j}$ in 3.2. and suppose the numbering is such that the first $\rho$ columns are linearly independent. If a quintic vanishes on $\left(x_{j}\right)_{1 \leq j \leq \rho}$, it will consequently vanish on all $\left(x_{j}\right)_{1 \leq j \leq \nu}$. Theorem A shows that the germ $\left(\mathcal{N}_{\nu}, X\right)$ is the transversal intersection of the hypersurfaces $H\left(x_{j}\right), 1 \leq j \leq \rho$, defined in a neighborhood of $X$ in $\mathscr{N} \subset \prod_{4}^{5}$ by requiring that the node of $X$ at $x_{j}$ deforms trivially as $X$ deforms with parameter space $(\mathscr{N}, X)$.

Now "drop", say: $x_{1}$, i.e. take (locally) only the transversal intersection:

$$
\bigcap_{2 \leq j \leq \rho} H\left(x_{j}\right) .
$$

A generic quintic $Y$ in this intersection (sufficiently close to $X$ ) will have per force less nodes than $X$ and $\rho(Y)=\rho-1$.

It follows that $\delta(Y)=\nu(Y)-\rho(Y) \leq \nu-\rho=\delta$, and an iteration of the argument yields:

Theorem B. Let $X$ be a nodal quintic threefold (resp. a double solid ramified over a nodal octic surface), with $\nu(X)$ nodes and defect $\rho(X)$. Then the range of $\nu$ over $\mathcal{N} \subset \Pi_{4}^{5}$ (resp. $\prod_{3}^{8}$ ) omits at most $\delta(X)$ integers in the interval $[0, \nu(X)]$.

\section{SOME EXAMPLES}

In order to derive concrete estimates from our Theorem B, we review several examples in which the number of nodes $\nu$ and the defect $\delta$ are known.

4.1. Consider first the case of nodal octic surfaces.

The a priori upper bound obtained by Miyoaka in [14] for the number of nodes is 174 , while the highest value produced in examples is 160 (cf. [12, 7]).

Werner [22, Ch. VI], computed the defect of several Čmutov-threefolds which are double solids ramified over explicitly given nodal octics. These yield for $(\nu, \delta)$ the following values: $(108,0),(123,3),(136,7)$, and $(144,9)$. 
Consequently, nodal octic surfaces with any number of nodes up to 108 do exist, while up to 144 at most 9 "gaps" may possibly appear.

4.2. Turning now to the case of nodal quintic threefolds, we have, on the one hand, Varchenko's bound in [20] allowing no more than 135 nodes; on the other hand, Hirzebruch's example [22] of a quintic with 126 nodes, obtained by homogenizing the equation:

$$
f(x, y)-f(u, v)=0
$$

with $f(x, y)=0$ the equation of a regular pentagon in the affine plane. Further investigations by Werner and van Geemen [23] have shown that small (or standard) resolutions of Hirzebruch's quintic are rigid, i.e. $\delta=25$.

Close to this, we have the quintic with 125 nodes defined by:

$$
x_{0}^{5}+x_{1}^{5}+x_{2}^{5}+x_{3}^{5}+x_{4}^{5}-5 x_{0} x_{1} x_{2} x_{3} x_{4}=0
$$

and considered by Schoen in [16]. Small resolutions are also rigid, so that $\delta=24(\rho=101, m=0)$.

Thus, up to 126, at most 24 "gaps" for $\nu$ might exist.

4.2.1. At this point we can answer a question raised by Schoen in [17] on the existence, for given $m, 0 \leq m \leq 101$, of $m$-dimensional families $H_{m}$ of nodal quintics, such that, for the general point $X \in H_{m}$, the map:

$$
T_{X}\left(H_{m}\right) \rightarrow H^{1}\left(X, \operatorname{Hom}\left(\Omega_{X}^{1}, \mathscr{O}_{X}\right)\right)
$$

be an isomorphism.

The positive answer now follows from 3.4. since

$$
H^{1}\left(X, \operatorname{Hom}\left(\Omega_{X}^{1}, \mathscr{O}_{X}\right)\right)=H^{1}\left(\widehat{X}, \Theta_{\widehat{X}}\right) .
$$

One starts with a rigid nodal quintic, shifting, for each increment $m \rightarrow m+1$ in the number of moduli, i.e. $\rho \rightarrow \rho-1$, to a nearby component of some $\mathscr{N}_{\nu}$ as in 3.5., where one takes (locally) a slice transverse to the orbits of $\operatorname{PGL}(5)$.

4.2.2. We record here some other values for $(\nu, \rho)$ resulting from examples and computations in [8]; namely, $(114,16),(118,18)$, and $(120,20)$.

In addition, we have:

Lemma 4.2.3. The dependency locus of five generic sections in $\mathscr{O}_{P_{4}}(1)^{5}$ is a quintic with 50 nodes and defect $\delta=1$.

Proof. That the generic case yields 50 nodes follows from the Thom-Porteous formula. For the defect, consider the bundle $F=\pi_{1}^{*} \mathscr{O}_{P_{4}}(1)^{5} \otimes \pi_{2}^{*} \mathscr{O}_{P_{4}}(1)$ over $P_{4} \times P_{4}$ with projections $\pi_{1}, \pi_{2}$, and note that

$$
H^{0}(F)=H^{0}\left(P_{4}, \pi_{1 *} F\right)=H^{0}\left(P_{4}, \mathscr{O}_{P_{4}}(1)^{5}\right)^{5} .
$$

The zero-locus $\hat{X}$ of a generic global section of $F$ will therefore project by $\pi_{1}$ on a quintic $X$ of the envisaged type, giving a small resolution of the latter. Clearly $b_{2}(\widehat{X})=2$, i.e. $\delta(X)=1$. 
Less immediate is the following:

Lemma 4.2.4. The dependency locus of two generic sections in the HorrocksMumford bundle over $P_{4}$ is a quintic with 100 nodes and defect $\delta=3$.

Proof. The first part has been already pointed out in [16]. To compute the defect, we look at the small resolution resulting in the generic case $X$ from the zero-locus $\widehat{X}$ of a section in $F=\pi_{1}^{*} E \otimes \pi_{2}^{*} \mathscr{O}_{P_{1}}(1)$ over $P_{4} \times P_{1}$, where $E$ stands for the Horrocks-Mumford bundle over $P_{4}$. In the long exact sequence:

$$
0 \rightarrow H^{0}\left(\Theta_{P_{4} \times P_{1}} \mid \widehat{X}\right) \rightarrow H^{0}(F \mid \widehat{X}) \rightarrow H^{1}\left(\Theta_{\widehat{X}}\right) \rightarrow H^{1}\left(\Theta_{P_{4} \times P_{1}} \mid \widehat{X}\right) \rightarrow \cdots
$$

we may compute, using the Koszul resolution for $\mathscr{O}_{\widehat{X}}$, the Leray spectral sequence and results on the cohomology of $E, E \otimes E^{*}$ etc. from [13]:

$$
h^{0}\left(\Theta_{P_{4} \times P_{1}} \mid \widehat{X}\right)=27, \quad h^{1}\left(\Theta_{P_{4} \times P_{1}} \mid \widehat{X}\right)=4, \quad h^{0}(F \mid \widehat{X}) \leq 31 .
$$

In the notations of $\S 3, h^{1}\left(\Theta_{\widehat{X}}\right)=m=\operatorname{dim}\left(\mathscr{N}_{100}, X\right)-24$.

Recall that the quintics under consideration are parametrized by an open set of the Grassmannian $G\left(2, H^{0}(E)\right)$ of 2-planes in $H^{0}(E)$, with $h^{0}(E)=4$. $G\left(2, H^{0}(E)\right)$ defines a four-dimensional quadric in the projective space given by quintic homogeneous polynomials invariant under the Heisenberg group $H_{5}$.

The linear space $W$ corresponding to the tangent space $T_{X}\left(\mathscr{N}_{100}\right)$ is necessarily an invariant space for $H_{5}$ and contains a five-dimensional subspace of invariant quintics. The above computations imply:

$$
\operatorname{dim} W \leq 25+(31-27)+4=33 .
$$

The decomposition of the space of quintics under $H_{5}$ (cf. [11]) and the overall symmetry under its normalizer now show this can only happen for $\operatorname{dim} W=5+24$, i.e. $m=4$. Since $e(X)=0$, this gives $\delta=3$.

\section{REFERENCES}

1. M. F. Atiyah, On analytic surfaces with double points, Proc. Roy Soc. London Ser. A 245 (1958), 237-244.

2. J. W. Bruce, On complex projective hypersurfaces, Proc. Edinburgh Math. Soc. 24 (1981), 91-97.

3. F. Catanese and G. Ceresa, Constructing sextic surfaces with a given number $d$ of nodes, J. Pure Appl. Algebra 23 (1982), 1-12.

4. H. Clemens, Double solids, Adv. in Math. 47 (1983), 107-230.

5. H. Flenner and S. Kosarew, On locally trivial deformations, Publ. Res. Inst. Math. Sci. 23 (1987), 627-665.

6. R. Friedman, Simultaneous resolution of threefold double points, Math. Ann. 274 (1986), 671-689.

7. D. Gallarati, Una superficie dell'ottavo ordine con 160 nodi, Acc. Ligure Sci. lett. 14 (1957), $1-7$.

8. B. van Geemen and J. Werner, Nodal quintics in $P^{4}$, preprint.

9. G. M. Greuel and U. Karras, Families of varieties with prescribed singularities, Compositio Math. 69 (1989), 83-110. 
10. F. Hirzebruch, Some examples of threefolds with trivial canonical bundle, Collected Papers II (no. 75), Springer-Verlag, Heidelberg, 1987, pp. 757-770.

11. K. Hulek, Projective geometry of elliptic curves, Astérisque, Soc. Math. de France, 1986.

12. O. Kreiss, Über syzygetische Flächen, Annali di Mat. 41 (1956), 105-111.

13. N. Manolache, On the normal bundle to Abelian surfaces embedded in $P^{4}(C)$, Manuscripta Math. 55 (1986), 111-119.

14. Y. Miyaoka, The maximal number of quotient singularities on surfaces with given numerical invariants, Math. Ann. 268 (1984), 159-171.

15. Ch. Schoen, Algebraic cycles on certain desingularized nodal hypersurfaces, Math. Ann. 279 (1985), 17-27.

16. - On the geometry of a special determinantal hypersurface associated to the MumfordHorrocks vector bundle, J. Reine Angew. Math. 364 (1986), 85-111.

17. _ On fibre products of rational elliptic surfaces with section, Math. Z. 197 (1988), 177199.

18. A. Strominger and E. Witten, New manifolds for superstring compactification, Comm. Math. Phys. 101 (1985), 341-361.

19. G. Tian, Smoothness of the universal deformation space of compact Calabi-Yau manifolds and its Peterson-Weil metric, Mathematical Aspects of String Theory (S. T. Yau, ed.), World Scientific, Singapore, 1987.

20. A. N. Varchenko, On semicontinuity of the spectrum and an upper bound for the number of singular points of projective hypersurfaces, Dokl. Akad. Nauk USSR 270 (1983). (Russian)

21. J. Wehler, Cyclic coverings: deformation and Torelli theorem, Math. Ann. 274 (1986), 443472.

22. J. Werner, Kleine Auflösungen spezieller dreidimensionaler Varietäten, Bonner Math. Schriften 186 (1987).

23. _ ( with an appendix by B. van Geemen), New examples of threefolds with $c_{1}=0$, preprint.

Institute for Advanced Study, Princeton, New Jersey 08540 